Acta Cardiologica

\title{
Serum albumin level and long-term outcome in acute heart failure
}

\section{Arnaud Ancion, Sophie Allepaerts, Sébastien Robinet, Cecile Oury, Luc A. Pierard \& Patrizio Lancellotti}

To cite this article: Arnaud Ancion, Sophie Allepaerts, Sébastien Robinet, Cecile Oury, Luc A. Pierard \& Patrizio Lancellotti (2019): Serum albumin level and long-term outcome in acute heart failure, Acta Cardiologica, DOI: 10.1080/00015385.2018.1521557

To link to this article: https://doi.org/10.1080/00015385.2018.1521557

曲 Published online: 16 Jan 2019.

Submit your article to this journal $\asymp$

山 Article views: 53

Q View related articles $₫$

View Crossmark data

Citing articles: 1 View citing articles 


\title{
Serum albumin level and long-term outcome in acute heart failure
}

\author{
Arnaud Ancion $^{\mathrm{a}}$, Sophie Allepaerts ${ }^{\mathrm{b}}$, Sébastien Robinet ${ }^{\mathrm{a}}$, Cecile Oury $^{\mathrm{a}}$ (D), Luc A. Pierard ${ }^{\mathrm{a}}$ and Patrizio \\ Lancellotti ${ }^{\mathrm{a}, \mathrm{c}}$ \\ aUniversity Hospital of Liège, GIGA Cardiovascular Sciences, Acute Care Unit, Heart Failure Clinic, CHU Sart Tilman, Liège, Belgium; \\ ${ }^{b}$ University Hospital of Liège, Geriatric Medicine, CHU Sart Tilman, Liège, Belgium; 'Gruppo Villa Maria Care and Research, Anthea \\ Hospital, Bari, Italy
}

\begin{abstract}
Objective: Hypoalbuminemia is common in heart failure (HF), especially in elderly patients. It is associated with an increased risk of death. The present study sought to examine the prognostic significance of serum albumin level in the prediction of long-term mortality in patients admitted for acute HF.

Methods and results: We examined the association between albumin and hospital mortality in a cohort of 509 patients admitted for acute HF. None of the patients had infectious disease, severe arrhythmias (atrial fibrillation, ventricular tachycardia, ventricular fibrillation), required invasive ventilation or presented with acute coronary syndrome or primary valvular disease. Sixty-nine patients (14\%) died during the 1-year follow-up. With multivariable analysis, haemoglobin level $(p=.003)$, systolic blood pressure $(p=.004)$ and serum albumin level $(p=.003)$ emerged as independent predictors of long-term mortality. Hypoalbuminemia $(<35.7 \mathrm{~g} / \mathrm{L})$ had a hazard ratio of $2.01(95 \% \mathrm{Cl} 1.24-3.25)$ and haemoglobin of 2.6 (95\% Cl 1.29-5.22) for predicting long-term mortality.

Conclusions: Serum albumin level measured at admission, especially if combined with anaemia, can serve as a simple prognostic factor in acute HF for predicting long-term outcome.
\end{abstract}

\section{ARTICLE HISTORY}

Received 3 August 2018

Revised 20 August 2018

Accepted 4 September 2018

\section{KEYWORDS}

Acute heart failure; albumin; long-term outcome

\section{Introduction}

Acute heart failure (HF) is a growing medical problem associated with major morbidity and mortality [1]. Options for the management of these patients remain limited with high in-hospital mortality rates. Accurate individual risk stratification can thus help physicians to choose the intensity of care needed and promote tailored medical decision-making [2].

Hypoalbuminemia is common in patients with $\mathrm{HF}$ [3], especially in the elderly likely in relation with the increasing rate of frailty [4]. In HF, hypoalbuminemia may be a marker of comorbidity burden, inflammatory state, malnutrition, and cachexia [5]. Low serum albumin levels are associated with increased risk of HF onset and progression. Indeed, hypoalbuminemia may promote pulmonary congestion, [6] myocardial oedema and subsequent worsening of myocardial dysfunction, [7] diuretic resistance and fluid retention, [8] and a decrease in antioxidant functions and antiinflammatory properties [9]. Several authors have demonstrated an association between low serum albumin and increased cardiovascular morbidity and mortality in patients with chronic HF [4,10-12]. Conversely, very few studies have examined the impact of hypoalbuminemia in the acute HF setting [12,13]. In elderly patients (>80 years) [14,15] and nonagenarians [16] with acute HF, severe hypoalbuminemia represents a potential predictor of adverse in-hospital outcome [17-20]. To our knowledge, the clinical relevance of hypoalbuminemia has not yet been fully evaluated in this clinical setting. The aim of the present study was to examine the prognostic significance of serum albumin level in the prediction of long-term outcome of patients admitted for acute non-ischaemic HF in a Belgian community hospital.

\section{Methods}

\section{Population}

The present study collected detailed hospitalisation data from computerised medical records of patients presenting with acute HF at $\mathrm{CHU}$ of Liège, Belgium,

CONTACT Patrizio Lancellotti plancellotti@chuliege.be Department of Cardiology, University Hospital, Université de Liège, CHU du Sart Tilman, 4000 Liège, Belgium

(C) 2019 Belgian Society of Cardiology 
between 2010 and 2012. Patients $(n=1611)$ were eligible for the first round of selection if they were $>18$ years of age, had a suspected diagnosis of HF and were alive $24-36 \mathrm{~h}$ after admission. After the second round of selection, 899 patients with $\geq 1$ following criteria were disqualified: respiratory support, cardiogenic shock, acute coronary syndrome, inotropic support, primary valvular heart disease, permanent pacemaker pacing, severe arrhythmias (atrial fibrillation, ventricular tachycardia, ventricular fibrillation), infectious/ inflammatory disease (C-reactive protein $>10 \mathrm{mg} / \mathrm{L}$ ), end-stage renal failure requiring dialysis, cancer. At the end of the selection process, we kept 509 patients with a measure of serum albumin level and long-term follow-up available. The study protocol conforms to the ethical guidelines of the 1975 Declaration of Helsinki as reflected in a priori approval by the institution's human research committee.

\section{Data collection}

The following clinical entry data were abstracted from hospital records: demographic information, the use of beta-blocker or angiotensin-converting enzyme (ACE) inhibitor at admission, medical history, prior myocardial infarction, prior HF hospitalisation, laboratory findings (haemoglobin, sodium, creatinine, NT-proBNP and albumin), heart rate, blood pressure, left ventricular ejection fraction. Serum albumin levels were analysed using a bromocresol purple dye-binding method. The in-house reference range for this albumin assay is $38-49 \mathrm{~g} / \mathrm{L}$, with a total imprecision $<2.5 \%$. All other blood tests were performed using standard routine techniques. The study population and method were described previously as the in-hospital outcome [17].

\section{Statistical methods}

Data are reported as mean \pm SD for continuous variables or percentages of patients for categorical variables. Quantitative variables were tested for distribution normality with the Shapiro-Wilk test. Group comparisons for categorical variables were obtained by a chi-square test and for continuous variables with Mann-Whitney-Wilcoxon test as they were not normally distributed. These groups were defined following two criteria, used in two distinct analyses: first, based on mortality and secondly, based on the appearance of a recurrent heart failure event during follow-up. Hazard ratios of the multiple Cox-regressions were log-transformed and results of these analyses were pooled following Rubin's rules with the
'Hmisc' and 'rms' R-packages (Harrel FE, Regression modelling strategies). Survival curves were computed based on the Kaplan-Meier method using the tertiles boundaries for albumin, haemoglobin and arterial systolic blood pressure. We also classified the patients into four categories considering that their albumin and haemoglobin values were abovelbelow the thresholds used in Kaplan-Meier curves. We compared the proportion of dead patients in these four categories using a Fisher's exact test and corrected our $p$-values for multiple testing following Holm's method. All statistical analyses were performed with $\mathrm{R}$ version 3.2.0 (R Foundation for Statistical Computing, Vienna, Austria). A $p$-value $<.05$ was considered statistically significant.

\section{Results}

\section{Patients' characteristics}

Among the 509 patients included $(72 \pm 12$ years, 58\% of male), $48 \%$ had hypertension, $19 \%$ were diabetics, $19 \%$ previously experienced myocardial infarction, $42 \%$ had ischaemic heart disease and $45 \%$ had prior heart failure diagnosis (Table 1). Left ventricular systolic dysfunction (ejection fraction $<40$ ) was identified in 227 (45\%) patients. Angiotensin-converting enzyme (ACE)-inhibitor and beta-blocker were taken at the time of admission by 170 and 186 patients, respectively.

\section{Outcome data}

During follow-up, 69 patients (14\%) died and 103 (20\%) developed a new heart failure event. The comparison of survivors and non-survivors are reported in Table 1. Patients who died had significantly lower albumin level $(p=.0003)$, haemoglobin $(p=.0004)$ and haematocrit levels $(p=.006)$ and arterial systolic $(p=.001)$ and diastolic blood pressure $(p=.006)$. Medical treatment was similar between groups. Similar data were observed in patients with recurrent heart failure, though these patients were younger $(p=.03)$ than those with uneventful. On the contrary, the albu$\min (p=.49)$, haemoglobin $(p=.35)$ and haematocrit $(p=.48)$ levels were similar in patients with or without recurrent heart failure.

\section{Predictors of long-term mortality}

Based on backward selection applied to the Coxregression model for mortality (including age, albumin and haemoglobin levels, arterial systolic and diastolic 
Table 1. Univariable analysis for potential predictors of mortality.

\begin{tabular}{|c|c|c|c|c|}
\hline Variables & Whole cohort $(n=509)$ & Survivors $(n=440,86 \%)$ & Death $(n=69,14 \%)$ & $p$ \\
\hline Age, years & $72 \pm 12$ & $72 \pm 12$ & $72 \pm 13$ & .53 \\
\hline Male gender, $n(\%)$ & $297(58)$ & $257(58)$ & $40(58)$ & .99 \\
\hline Heart rate, bpm & $79 \pm 18$ & $78 \pm 18$ & $80 \pm 16$ & .29 \\
\hline Systolic blood pressure, $\mathrm{mmHg}$ & $123 \pm 21$ & $124 \pm 21$ & $114 \pm 20$ & .001 \\
\hline Diastolic blood pressure, $\mathrm{mmHg}$ & $69 \pm 12$ & $69 \pm 12$ & $64 \pm 12$ & .006 \\
\hline Left ventricular ejection fraction, \% & $45 \pm 16$ & $45 \pm 16$ & $42 \pm 16$ & .10 \\
\hline Left ventricular ejection fraction $<40 \%, n(\%)$ & $227(45)$ & $190(43)$ & $37(54)$ & .35 \\
\hline \multicolumn{5}{|l|}{ Medical history } \\
\hline Hypertension, $n(\%)$ & $246(48)$ & $210(48)$ & $36(52)$ & .75 \\
\hline Diabetes, $n(\%)$ & 97 (19) & 85 (19) & $12(17)$ & .74 \\
\hline COPD, $n(\%)$ & $126(25)$ & $102(23)$ & $24(35)$ & .07 \\
\hline Ischaemic cardiomyopathy, $n$ (\%) & $213(42)$ & $177(40)$ & $36(52)$ & .08 \\
\hline Prior cardiac decompensation, $n(\%)$ & $227(45)$ & $190(43)$ & $37(54)$ & .14 \\
\hline Prior myocardial infarction, $n(\%)$ & $98(19)$ & $80(18)$ & $18(26)$ & .17 \\
\hline \multicolumn{5}{|l|}{ Medications } \\
\hline ACE-inhibitor & $170(33)$ & $147(33)$ & $23(33)$ & 1 \\
\hline Beta-blockers & $186(37)$ & $161(37)$ & $25(36)$ & .99 \\
\hline \multicolumn{5}{|l|}{ Laboratory findings } \\
\hline Total cholesterol & $1.4 \pm 0.4$ & $1.5 \pm 0.4$ & $1.4 \pm 0.4$ & .31 \\
\hline Triglycerides, $\mathrm{mmol} / \mathrm{L}$ & $0.96 \pm 0.48$ & $0.97 \pm 0.5$ & $0.91 \pm 0.37$ & .88 \\
\hline Glycemia, g/L & $1.2 \pm 0.5$ & $1.2 \pm 0.5$ & $1.1 \pm 0.4$ & .68 \\
\hline Haematocrite, \% & $37 \pm 6$ & $38 \pm 6$ & $35 \pm 6$ & .006 \\
\hline Haemoglobin, g/dL & $12.1 \pm 2.2$ & $12.3 \pm 2.1$ & $11.1 \pm 2.1$ & .0004 \\
\hline White blood cells count, $\%$ & $8.3 \pm 3.9$ & $8.4 \pm 4$ & $7.5 \pm 2.8$ & .08 \\
\hline Albumin, $\mathrm{g} / \mathrm{L}$ & $36.9 \pm 4.1$ & $37.2 \pm 4.1$ & $35.2 \pm 4.2$ & .0003 \\
\hline Total protein, g/L & $64.8 \pm 6.3$ & $64.8 \pm 6.3$ & $65.1 \pm 6.3$ & .74 \\
\hline NT-proBNP, pg/ml & $8354 \pm 10673$ & $7362 \pm 7631$ & $15552 \pm 22257$ & .048 \\
\hline Calcium, mmol/L & $2.2 \pm 0.1$ & $2.2 \pm 0.1$ & $2.2 \pm 0.1$ & .69 \\
\hline Sodium, $\mathrm{mmol} / \mathrm{L}$ & $140.7 \pm 4.3$ & $140.7 \pm 4.4$ & $140.8 \pm 3.5$ & .81 \\
\hline Creatinine, mg/dl & $14.4 \pm 10.8$ & $13.7 \pm 10.2$ & $18.1 \pm 13.5$ & .002 \\
\hline Estimated GFR, $\mathrm{ml} / \mathrm{min} / \mathrm{mm}^{3}$ & $57.8 \pm 26.6$ & $59.3 \pm 26.3$ & $48.7 \pm 26.9$ & .002 \\
\hline TGO, UI/L & $42.6 \pm 129.4$ & $40.2 \pm 129.9$ & $56.2 \pm 126.6$ & .01 \\
\hline TGP, UI/L & $40.9 \pm 121.1$ & $38.5 \pm 117.2$ & $55.3 \pm 142.4$ & .48 \\
\hline
\end{tabular}

Table 2. Multivariable cox-regression analysis for mortality.

\begin{tabular}{lccc}
\hline Variable & OR & $95 \% \mathrm{Cl}$ & $p$ \\
\hline Albumin & 1.09 & $1.03-1.15$ & .003 \\
Haemoglobin & 1.21 & $1.07-1.37$ & .003 \\
Arterial systolic blood pressure & 1.02 & $1.01-1.04$ & .004 \\
\hline
\end{tabular}

OR for albumin, haemoglobin and arterial systolic blood pressure are expressed relative to a decreased in their values.

blood pressure, creatinine, and NT-proBNP levels), albumin level $(p=.003)$, haemoglobin level $(p=.003)$ and arterial systolic blood pressure $(p=.004)$ had significant prognostic effects on mortality (Table 2). In the multivariable cox-regression analysis described in Table 2, the adjusted hazard ratios (HR) for mortality were 1.09 (95\% Cl: $1.03-1.15)$ and 1.21 (95\% Cl: 1.07-1.37) for a decrease of $1 \mathrm{~g} / \mathrm{L}$ of either albumin or haemoglobin value, respectively. Adjusted HR for mortality was 1.02 (95\% Cl: 1.01-1.04) per decrease of $1 \mathrm{mmHg}$ in arterial systolic blood pressure.

Figure 1 shows the Kaplan-Meier curves for albumin (A), haemoglobin (B) and arterial systolic blood pressure (C) using the tertiles boundaries as the threshold for classification of the subpopulations as this improved the distinction between classes according to the Log-rank tests (Figure 1). The remaining survival probabilities showed that patients with $\geq 35.7 \mathrm{~g} \backslash \mathrm{L}$ of albumin as well as those with $\geq 12.1 \mathrm{~g} \backslash \mathrm{dL}$ of haemoglobin at admission time had a better outcome than the others. In a lesser extent, having an arterial systolic blood pressure $\geq 120 \mathrm{mmHg}$ was also associated with a better prognosis.

The only variable for which we found a prognostic effect for heart failure recurrence was the arterial systolic blood pressure $(p=.006$, data not shown) (according to backward selection on a Cox-regression model including arterial systolic and diastolic blood pressure, history of myocardial infraction or cardiac decompensation, ejection fraction, cholesterol level and age).

\section{Prognostic effect of albumin and haemoglobin level}

In a Cox-regression model adjusted for arterial systolic blood pressure, we tested the effect of having an albumin level $<35.7 \mathrm{~g} \backslash \mathrm{L}$ or a haemoglobin level $<12.1 \mathrm{~g} \backslash \mathrm{dL}$ on mortality during follow-up (Table 3). Adjusted HRs were 2.01 (95\% Cl: 1.24-3.25) and 2.6 (95\% Cl: 1.29-5.22) for patients with an albumin level $<35.7 \mathrm{~g} \backslash \mathrm{L}$ or haemoglobin level $<12.1 \mathrm{~g} \backslash \mathrm{dL}$, respectively

We then assessed our 509 individuals to four categories based on their albumin and haemoglobin level 

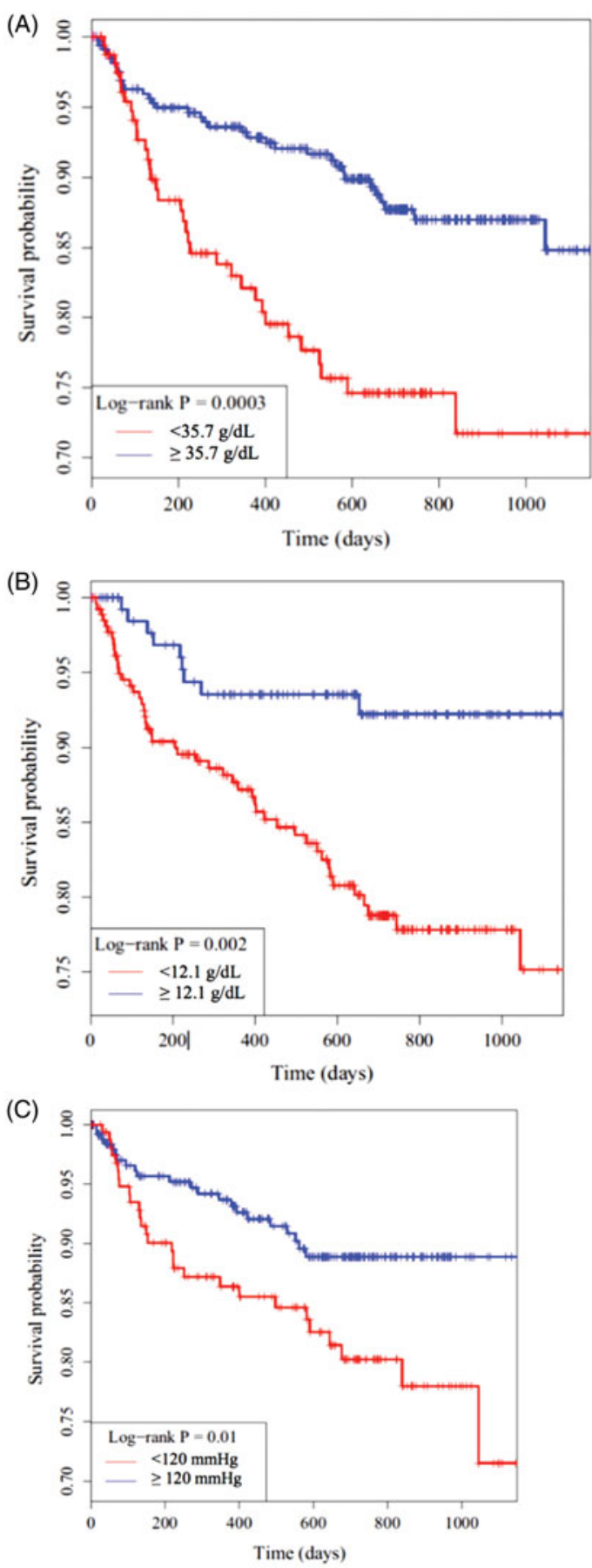

Figure 1. Kaplan-Meier Curves stratified by albumin level $(A)$, haemoglobin level (B) and arterial systolic blood pressure (C).

as follow: individuals with albumin $\geq 35.1 \mathrm{~g} \mathrm{LL}$ and haemoglobin $\geq 12.1 \mathrm{~g} \backslash \mathrm{L}$ (category 1), individuals with albumin $\geq 35.1 \mathrm{~g} \backslash \mathrm{L}$ and haemoglobin $<12.1 \mathrm{~g} \backslash \mathrm{dL}$ (category 2), individuals with albumin $<35.1 \mathrm{~g} \backslash \mathrm{L}$ and haemoglobin $\geq 12.1$ g $\backslash d L$ (category 3 ), individuals with albumin $<35.1 \mathrm{glL}$ and haemoglobin $<12.1 \mathrm{gldL}$ (category 4). The proportion of survivors and deaths in these categories are reported in Table 4 and Figure 2. We found that the risk of mortality was significantly
Table 3. Cox-regression analysis for mortality with threshold for albumin and haemoglobin.

\begin{tabular}{lccl}
\hline Variable & OR & $95 \% \mathrm{Cl}$ & $p$ \\
\hline Albumin $<35.7 \mathrm{g \backslash L}$ & 2.01 & $1.24-3.25$ & .005 \\
Haemoglobin $<12.1 \mathrm{~g} \backslash \mathrm{dL}$ & 2.60 & $1.29-5.22$ & .01 \\
Arterial systolic blood pressure $(<120 \mathrm{mmHg})$ & 1.02 & $1.01-1.04$ & .003 \\
\hline
\end{tabular}

Table 4. Contingency table for the proportion of survivors and deaths in the four categories defined albumin and haemoglobin levels.

\begin{tabular}{lcc}
\hline Category & Survivors (\%) & Deaths (\%) \\
\hline 1 & $99(95.2)$ & $5(4.8)$ \\
2 & $134(83.8)$ & $26(16.3)$ \\
3 & $33(90)$ & $7(17.5)$ \\
4 & $88(80)$ & $22(20)$ \\
\hline
\end{tabular}

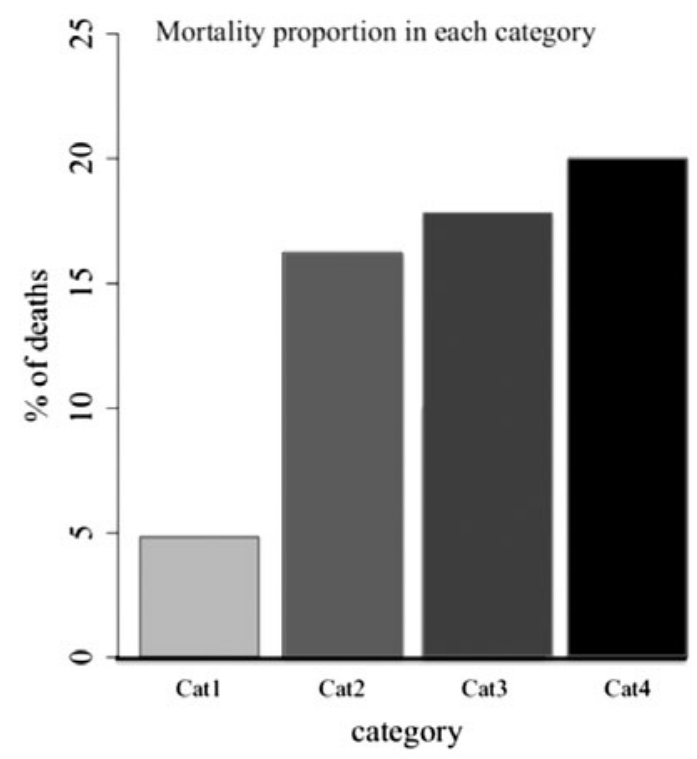

Figure 2. Proportion of dead patients in each category.

different between category 1 and $4\left(p_{\text {cat1-4 }}=0.001\right)$ and between category 1 and $2\left(p_{\text {cat } 1-2}=0.01\right)$. So, a significant increase in mortality was observed in categories, which are defined by the haemoglobin level $<12.1 \mathrm{~g} \backslash \mathrm{L}$. Indeed, the proportion of deaths was similar between category 1 and 3 although the third category is defined by a drop in albumin level (albumin $<35.7 \mathrm{~g} \backslash \mathrm{L}$, haemoglobin $\geq 12.1 \mathrm{~g} \backslash \mathrm{dL}$ ).

\section{Discussion}

Our data demonstrate that hypoalbuminemia is not only common in patients with acute HF but predicts long-term outcome. Low serum albumin level is associated with significantly increased all-cause mortality but not with recurrent heart failure. Decreased haemoglobin level and systolic blood pressure were additional predictive factors. 


\section{Long-term outcome predictors in acute HF}

Several clinical (older age, severe symptoms, systolic blood pressure) and biochemical (blood urea nitrogen, serum creatinine and natriuretic peptides) markers have been recently proposed for screening at presentation patients with acute HF syndromes at risk for inhospital death and long-term outcome. Recently, we, as others, reported that hypoalbuminemia represents a simplistic factor associated with an increased risk of in-hospital mortality. Hypoalbuminemia is a marker of ageing, malnutrition and inflammation, and more broadly of cachexia related to the severity and chronicity of HF. In the acute HF setting, fluid retention, increased vascular permeability, impaired liver synthesis and renal losses may also contribute to further decrease in serum albumin level. We already showed that in the setting of acute HF, lower levels of albumin were related to ageing, lower haemoglobin (multifactorial cause) and cholesterol (malnutrition/inflammation) levels, impaired renal and hepatic function, lower rate of beta-blockers and ACE inhibitors use.

In the present study, we examined the long-term outcome of our series of patients admitted for acute $\mathrm{HF}$ and showed a $14 \%$ 1-year mortality rate close to previous registries with similar rate of comorbidities [18-24]. Hypoalbuminemia, anaemia and lower systolic blood pressure at admission, all three reflecting different pathophysiologic aspects of HF emerged as independent predictors of long-term mortality. Albumin has already been suggested as a potential predictor of short-term mortality in other studies, but often embedded in complex nutrition scores $[18,19]$. The presence of hypoalbuminemia reflects an advanced disease process with significant metabolic repercussions, even in the acute setting phase. The long-term survival of patients with low serum albumin levels $(<35.7 \mathrm{~g} \backslash \mathrm{L})$ was markedly decreased. The risk of deaths was multiplied by about 2 and even by 4 in the higher tertile. Noteworthy, this threshold was similar to the one reported in the CONUT and the GNRI scores $[18,19]$.

Anaemia is a common comorbidity in $\mathrm{HF}$, which is associated with more symptoms, increased rate of hospitalisation and increased mortality [1]. The aetiology of anaemia is multifactorial, complex, and varies between patients. In HF, anaemia is considered to develop due to a complex interaction of iron deficiency, kidney disease and cytokine production, although micronutrient insufficiency and blood loss may also contribute $[25,26]$. All these factors are almost those associated with decreased serum albumin levels. In our study, a level of haemoglobin
$<12.1 \mathrm{~g} / \mathrm{dL}$, defining anaemia, represented an independent risk factor associated with increased longterm mortality. Interestingly, when anaemia was combined with hypoalbuminemia, patients displayed the lowest survival rates.

Lower systolic blood pressure was also pointed out to increase decrease the long-term survival, confirming previous observations. Post-hoc analysis of PARADIGMHF study demonstrated increased mortality and cardiovascular events in patients with the lowest blood pressure levels [27]. Lower blood pressure is considered as a sign of a more severe illness. In HF, the severity of the disease can be extrapolated by measuring the drug dosage (ACE, Beta-blocker) that patients are able to tolerate. The less they tolerate the treatments, the worse their prognosis. Recent works have shown that patients who tolerate less than $50 \%$ of the recommended doses of ACE-inhibitors or beta-blockers have an increased risk of death and hospitalisation for HF [28].

\section{Clinical implications}

Anaemia and hypoalbuminemia are simple, easily accessible and inexpensive prognostic factors in patients with acute HF. Their outcome impact is far beyond the acute stage, being able to predict the long-term mortality. Then, patients with low serum albumin levels or anaemia should receive more aggressive therapeutic support while targeting their causes. Although the precise cut-off to define anaemia in HF has mostly been arbitrary, its management has recently been well codified [1]. The use of intravenous iron has been shown to be associated with significant improvements in maximal exercise capacity and is accompanied by improved symptoms [29].

On the contrary, the management of patients with low serum albumin levels is far from well established. The European recommendations clearly describe the concepts of sarcopenia or cardiac cachexia but do not offer special care because of the lack of evidence on this matter [1]. Nutritional interventions in hospitalised patients remain complex. A recent Cochrane review found no clear benefit of nutritional care for patients hospitalised with HF [30]. The populations and interventions studied were, however, very heterogeneous. The randomised PICNIC study demonstrated that a personalised and prolonged intervention reduced the risk of death and hospitalisation for HF [31]. These data, although based on a small population, should encourage intensifying the nutritional management of patients with HF both during the hospital stay and during the follow-up. 


\section{Limitations}

Our study has several limitations related to its retrospective nature that are worth noting. They do not pertain to all patients presenting with acute HF. Anthropometric data (weight, height, and body mass index), white blood cell components (neutrophils, lymphocytes, monocytes), nutritional status, and information about the use of additional HF treatment (mineralocorticoid receptor antagonist) were missing. However, the hyperhydration state of our HF patients makes the interpretation of anthropometric measurements very difficult. Specific inflammatory biomarkers (high sensitivity C-reactive protein, interleukins, tumour necrosis factor-a) were also not available. The potential association between albumin level and inflammatory status needs additional exploration. Before 2012, NTproBNP was not routinely measured, resulting in small number of patients with these data available. NTproBNP was then not included in the multivariable model. This low rate of NT-proBNP assay is found in Chan's retrospective study conducted at the same time [21]. The prognostic impact of albumin in these patients remains to be determined in further studies.

\section{Conclusions}

In acute HF, hypoalbuminemia is associated with lower long-term survival rate, especially when combined with anaemia and low systolic blood pressure. These data determine a profile of patients at higher risk who need to be treated more aggressively during the acute stage. They might require a closer follow-up. Our data need to be confirmed prospectively and merit further investigations.

\section{Acknowledgments}

The authors would like to thank CHU Sart Tilman Liège IT/ DMI team, Belgium for their support.

\section{Disclosure statement}

No potential conflict of interest was reported by the authors.

\section{ORCID}

Cecile Oury (D) http://orcid.org/0000-0002-7561-0132

\section{References}

[1] Ponikowski P, Voors AA, Anker SD, et al. 2016 ESC Guidelines for the diagnosis and treatment of acute and chronic heart failure: the task force for the diagnosis and treatment of acute and chronic heart failure of the European Society of Cardiology (ESC)Developed with the special contribution of the Heart Failure Association (HFA) of the ESC. Eur Heart J. 2016;37:2129-2200.

[2] Fonarow GC, Adams KF, Jr., Abraham WT, et al. Risk stratification for in-hospital mortality in acutely decompensated heart failure: classification and regression tree analysis. JAMA. 2005;293:572-580.

[3] Pasini E, Aquilani R, Gheorghiade $M$, et al. Malnutrition, muscle wasting and cachexia in chronic heart failure: the nutritional approach. Ital Heart J. 2003;4:232-235.

[4] Novack V, Pencina M, Zahger D, et al. Routine laboratory results and thirty day and one-year mortality risk following hospitalization with acute decompensated heart failure. PLoS One. 2010;5:e12184.

[5] Araujo JP, Lourenco P, Rocha-Goncalves F, et al. Nutritional markers and prognosis in cardiac cachexia. Int J Cardiol. 2011;146:359-363.

[6] Arques S, Ambrosi P. Human serum albumin in the clinical syndrome of heart failure. J Card Fail. 2011;17: 451-458.

[7] Dongaonkar RM, Stewart RH, Geissler $\mathrm{HJ}$, et al. Myocardial microvascular permeability, interstitial oedema, and compromised cardiac function. Cardiovasc Res. 2010;87:331-339.

[8] Elwell RJ, Spencer AP, Eisele G. Combined furosemide and human albumin treatment for diuretic-resistant edema. Ann Pharmacother. 2003;37:695-700.

[9] Roche M, Rondeau P, Singh NR, et al. The antioxidant properties of serum albumin. FEBS Lett. 2008;582: 1783-1787.

[10] Liu M, Chan CP, Yan BP, et al. Albumin levels predict survival in patients with heart failure and preserved ejection fraction. Eur J Heart Fail. 2012;14:39-44.

[11] Metra M, Cotter G, El-Khorazaty J, et al. Acute heart failure in the elderly: differences in clinical characteristics, outcomes, and prognostic factors in the VERITAS Study. J Card Fail. 2015;21:179-188.

[12] Uthamalingam S, Kandala J, Selvaraj V, et al. Outcomes of patients with acute decompensated heart failure managed by cardiologists versus noncardiologists. Am J Cardiol. 2015;115:466-471.

[13] Arques S, Roux E, Sbragia P, et al. Usefulness of serum albumin concentration for in-hospital risk stratification in frail, elderly patients with acute heart failure. Insights from a prospective, monocenter study. Int J Cardiol. 2008;125:265-267.

[14] Arques S, Roux E, Stolidi P, et al. Usefulness of serum albumin and serum total cholesterol in the prediction of hospital death in older patients with severe, acute heart failure. Arch Cardiovasc Dis. 2011;104:502-508.

[15] Arques S, Ambrosi P, Gelisse R, et al. Hypoalbuminemia in elderly patients with acute diastolic heart failure. J Am Coll Cardiol. 2003;42: 712-716.

[16] Yanagisawa S, Miki K, Yasuda N, et al. Clinical outcomes and prognostic factor for acute heart failure in nonagenarians: impact of hypoalbuminemia on mortality. Int J Cardiol. 2010;145:574-576. 
[17] Ancion A, Allepaerts S, Oury C, et al. Serum albumin level and hospital mortality in acute non-ischemic heart failure. ESC Heart Fail. 2017;4:138-145.

[18] Iwakami N, Nagai T, Furukawa TA, et al. Prognostic value of malnutrition assessed by controlling nutritional status score for long-term mortality in patients with acute heart failure. Int J Cardiol. 2017;230: 529-536.

[19] Honda Y, Nagai T, Iwakami N, et al. Usefulness of geriatric nutritional risk index for assessing nutritional status and its prognostic impact in patients aged $>/=65$ years with acute heart failure. Am J Cardiol. 2016;118: 550-555.

[20] Agra Bermejo RM, Gonzalez Ferreiro R, Varela Roman $A$, et al. Nutritional status is related to heart failure severity and hospital readmissions in acute heart failure. Int J Cardiol. 2017;230:108-114.

[21] Chan WW, Waltman Johnson K, Friedman HS, et al. Association between cardiac, renal, and hepatic biomarkers and outcomes in patients with acute heart failure. Hosp Pract (1995). 2016;44:138-145.

[22] Fujino $M$, Takahama $H$, Hamasaki $T$, et al. Risk stratification based on nutritional screening on admission: Three-year clinical outcomes in hospitalized patients with acute heart failure syndrome. J Cardiol. 2016;68: 392-398.

[23] Lancellotti P, Ancion A, Magne J, et al. Elevated heart rate at 24-36h after admission and in-hospital mortality in acute in non-arrhythmic heart failure. Int J Cardiol. 2015;182:426-430.

[24] Cheng $Y$, Shao $Y$, Weir CR, et al. Predicting adverse outcomes in heart failure patients using different frailty status measures. Stud Health Technol Inform. 2017;245:327-331.

[25] Jankowska EA, Wojtas K, Kasztura M, et al. Bone marrow iron depletion is common in patients with coronary artery disease. Int J Cardiol. 2015;182:517-522.

[26] Ishigami J, Grams ME, Naik RP, et al. Hemoglobin, albuminuria, and kidney function in cardiovascular risk: the ARIC (Atherosclerosis Risk in Communities) study. J Am Heart Assoc. 2018;7:e007209.

[27] Bohm M, Young R, Jhund PS, et al. Systolic blood pressure, cardiovascular outcomes and efficacy and safety of sacubitril/valsartan (LCZ696) in patients with chronic heart failure and reduced ejection fraction: results from PARADIGM-HF. Eur Heart J. 2017;38: 1132-1143.

[28] Ouwerkerk W, Voors AA, Anker SD, et al. Determinants and clinical outcome of uptitration of ACE-inhibitors and beta-blockers in patients with heart failure: a prospective European study. Eur Heart J. 2017;38:1883-1890.

[29] Houston BL, Hurrie D, Graham J, et al. Efficacy of iron supplementation on fatigue and physical capacity in non-anaemic iron-deficient adults: a systematic review of randomised controlled trials. BMJ Open. 2018;8: e019240.

[30] Feinberg J, Nielsen EE, Korang SK, et al. Nutrition support in hospitalised adults at nutritional risk. Cochrane Database Syst Rev. 2017;(5):CD011598.

[31] Bonilla-Palomas JL, Gamez-Lopez AL, CastilloDominguez JC, et al. Nutritional intervention in malnourished hospitalized patients with heart failure. Arch Med Res. 2016;47:535-540. 